\title{
Prism-based tunable InGaN/GaN self-injection locked blue laser diode system: study of temperature, injection ratio, and stability
}

\author{
Mohammed Z. M. Khan, , ${ }^{\text {a* Sani Mukhtar, }{ }^{\text {a }} \text { Jorge A. Holguín-Lerma, }}$ \\ Omar Alkhazragi, ${ }^{\mathrm{b}}$ Islam Ashry, ${ }^{\mathrm{b}}$ Tien $\mathrm{K}$. Ng, ${ }^{\mathrm{b}}$ and Boon S. Ooi ${ }^{\mathrm{b}}$ \\ ${ }^{a}$ King Fahd University of Petroleum and Minerals, Optoelectronics Research Laboratory, \\ Electrical Engineering Department, Dhahran, Saudi Arabia \\ ${ }^{b}$ King Abdullah University of Science and Technology, Photonics Laboratory, Computer, \\ Electrical, and Mathematical Sciences and Engineering Division, Thuwal, Saudi Arabia
}

\begin{abstract}
A quasicontinuously wavelength tuned self-injection locked blue laser diode system employing a prism is presented. A rigorous analysis of the injection ratio (IR) in the form of three systems, namely high (HRS, $\sim-0.7 \mathrm{~dB}$ IR), medium (MRS, $\sim 1.5 \mathrm{~dB}$ IR), and low (LRS, $\sim-3.0 \mathrm{~dB}$ IR) reflection systems, showed a direct relationship with the wavelength tunability whereas the usable system power exhibited an inverse correlation. In particular, MRS configuration demonstrated a concurrent optimization of tuning window and system power, thus emerging as a highly attractive candidate for practical realization. Moreover, a comprehensive investigation on two distinct MRS configurations employing different commercially available InGaN/GaN blue lasers, i.e., MRS-1 and MRS-2, displayed a wavelength tunability (system power) of $\sim 8.2 \mathrm{~nm}(\sim 7.6 \mathrm{~mW})$ and $\sim 6.3 \mathrm{~nm}(\sim 11.6 \mathrm{~mW})$, respectively, at a low injection current of $130 \mathrm{~mA}$. In addition, both MRS configurations maintained high-performance characteristic with corresponding average optical linewidths of $\sim 80$ and $\sim 58 \mathrm{pm}$ and a side-modesuppression-ratio of $\geq 12 \mathrm{~dB}$. Lastly, a thorough stability analysis of HRS and MRS configurations, which are more prone to system instabilities due to elevated IRs, is performed at critical operation conditions of a high injection current of $\geq 260 \mathrm{~mA}$ and a temperature of $40^{\circ} \mathrm{C}$, showing an extended stable performance of over 120 min, thus further substantiating the promising features of the prism-based systems for practical applications. (C) 2020 Society of Photo-Optical Instrumentation Engineers (SPIE) [DOI: 10.1117/1.JNP.14.036001]
\end{abstract}

Keywords: InGaN/GaN blue laser; tunable laser; self-injection locking; injection ratio; external cavity system.

Paper 20049 received May 6, 2020; accepted for publication Jun. 17, 2020; published online Jul. 2, 2020.

\section{Introduction}

InGaN/GaN edge-emitting semiconductor laser diodes (LDs) emitting in the blue region of the electromagnetic spectrum are indispensable light sources in a multitude of applications, such as frequency conversion, trace matter detection, spectroscopy, laser cooling, biomedical applications, etc. ${ }^{1-6}$ The popularity of LDs in such applications is due to their cost-effectiveness, simplicity, and small footprint as compared with other types of lasers, such as gas lasers, solid-state lasers, and others. In general, free-running blue LDs lases with multilongitudinal modes that are fundamentally dictated by the LD active region gain profile and cavity length. ${ }^{7}$ Hence, despite providing several benefits, the inherent multimode lasing emission poses limits to their potential deployment and thus restricts their competition with other laser types that are typically bulky and complex. Hitherto, there are several reports in the literature on improving the spectral purity of these InGaN/GaN LDs to near single-mode wavelength operation; for instance, distributed feedback gratings have been engaged to demonstrate near-single mode emission with high

*Address all correspondence to Mohammed Z. M. Khan, E-mail: zahedmk@kfupm.edu.sa

$1934-2608 / 2020 / \$ 28.00$ (C) 2020 SPIE 
side-mode-suppression-ratio (SMSR) ${ }^{8-10}$ However, this involves additional fabrication complexity in addition to not being commercially available yet.

Alternatively, external cavity configurations utilizing semiconductor LDs have been reported. They not only assisted in achieving extreme narrow-linewidth (single-mode) operation but also enabled wavelength tunability, thereby opening a new paradigm of realizing tunable visible semiconductor LDs systems, commonly known as Littrow or Littman external cavity diode laser system (ECDL). In such configurations, a wavelength filtering element in the form of dielectric gratings is deployed to diffract the optical feedback laser light back into the LD active region to narrow the emission spectrum as well as for wavelength tuning purpose, which is accomplished by rotating the motor-driven diffraction grating stage ${ }^{11-13}$ In this regard, a narrow linewidth of $\sim 20 \mathrm{pm}$ and SMSR of $\sim 40 \mathrm{~dB}$ with about 3-nm tunability has been reported in Ref. 11 on a high power 445-nm blue LD. While exploiting different types of gratings and studying their effects on the blue wavelength tunability and output power in the Littrow ECDL configuration, an overall 6-nm tuning span with an output power of $80 \mathrm{~mW}$, spectral linewidth ( $\Delta \lambda$, measured as full-width at half maximum) of $20 \mathrm{pm}$, and SMSR $>20 \mathrm{~dB}$ were achieved. However, an inverse relationship between the tuning window and usable output power in which the tunability reduced to $1.4 \mathrm{~nm}$ with a usable power of $530 \mathrm{~mW}^{13}$ has been observed. Moreover, in a similar system in Ref. 12, the tuning span was improved to $7.2 \mathrm{~nm}$ with $\Delta \lambda \sim 100 \mathrm{pm}$, SMSR as high as $\sim 35 \mathrm{~dB}$, and output power of about $10 \mathrm{~mW}$, whereas, after optimizing the output power to $1400 \mathrm{~mW}$, the tuning window reduced to $3.6 \mathrm{~nm}$. Later, in a bid to investigate the effect of the grating parameters on the blue Littrow ECDL system, a tuning span of $\sim 10.9 \mathrm{~nm}$ with about 3-mW usable power and an SMSR of 10 to $20 \mathrm{~dB}$ was achieved. Subsequently, a drop (increase) in tunability (output power) of $8.3 \mathrm{~nm}(500 \mathrm{~mW})$ and $3.6 \mathrm{~nm}(1200 \mathrm{~mW})$ at different grating parameters was achieved. ${ }^{14}$ In addition, in further exploiting this configuration, a single longitudinal mode operation as well as $4.3 \mathrm{~nm}$ wavelength tunability on a 445-nm blue LD was reported in Ref. 15, with a measured linewidth of $4.7 \mathrm{MHz}$ and output power of $1 \mathrm{~mW}$ at a low injection current, whereas at a high injection current the tuning range drops to $2.6 \mathrm{~nm}$ with an output power of about $17 \mathrm{~mW}$.

Recently, a competing self-injection locked (SIL) ECDL system was proposed and demonstrated by our group as a promising alternative to the existing Littrow ECDL system. ${ }^{16,17}$ The flexibility of this configuration was demonstrated by a filter-less wavelength tuning operation in the $\sim 532-\mathrm{nm}$ green region as well as, very recently, via a prism assisted SIL-ECDL system in the blue region. In the latter case, a record wideband wavelength tunability of $12.1 \mathrm{~nm}$ with $\sim 3-\mathrm{mW}$ usable power was reported while maintaining $\Delta \lambda \leq 110 \mathrm{pm}$ and SMSR as high as $40 \mathrm{~dB}$. This was demonstrated from the high reflection system (HRS) configuration with $\sim-0.7 \mathrm{~dB}$ IR (i.e., the ratio of the optical feedback power to the free-running laser power, measured at the laser front facet) at above the threshold current of $130 \mathrm{~mA}$. Moreover, at a higher injection current of $390 \mathrm{~mA}$, the HRS configuration asserted the system power and wavelength tunability inverse relation with demonstrated values of $24 \mathrm{~mW}$ and $\sim 7.6 \mathrm{~nm}$, respectively. On the other hand, decreasing the IR to $\sim-3.0 \mathrm{~dB}$, i.e., a low reflection system (LRS), a high usable power of $\sim 15(\sim 180) \mathrm{mW}$ and a tunability of $\sim 7.0(\sim 4.5) \mathrm{nm}$ at 130 (390) $\mathrm{mA}$ were achieved. Strikingly, both systems were stable and maintained $\Delta \lambda \leq 180 \mathrm{pm}$ and SMSR $\geq 10 \mathrm{~dB}$ without employing a precision wavelength filtering element such as diffraction gratings, even at high injection currents and temperatures at which injection locking is challenging to sustain. These attractive features, together with a simple design, make the SIL-ECDL tunable system a promising candidate in a multitude of applications. However, as inferred from the above discussion, both HRS and LRS configurations are unable to optimize system power-wavelength tunability concurrently, which is a highly desired attribute of tunable lasers.

In this work, we propose and demonstrate a medium optical feedback prism-based blue SIL-ECDL tunable system. A rigorous analysis in terms of injection current, temperature, and external cavity length is performed on a medium reflection system (MRS) configuration with IR $\sim-1.5 \mathrm{~dB}$ exhibiting a quasicontinuous tunability of $\sim 8.2(\sim 5.6) \mathrm{nm}$ with a system power of $\sim 7.6(\sim 110) \mathrm{mW}$ at $130(390) \mathrm{mA}$, while maintaining an average $\Delta \lambda$ and SMSR of $\sim 80(\sim 155) \mathrm{pm}$ and $\sim 22.5(\sim 20) \mathrm{dB}$, respectively. Later, the effect of IR, in the form of HRS, MRS, and LRS configurations, on the performance of the prism-based blue SIL-ECDL system is 
comprehensively analyzed, uncovering the MRS configuration to be a more promising tunable laser source. In this case, a simultaneously optimized system power-wavelength tunability is achieved without compromising the $\Delta \lambda$ or SMSR characteristics. Furthermore, the effect of incorporating different commercial blue LDs on the performance of the MRS configuration is also examined wherein $\Delta \lambda$ of 37 to $79 \mathrm{pm}$ (average $\sim 58 \mathrm{pm}$ ) and tunability of $\sim 6.3 \mathrm{~nm}$ are measured with an improved estimated usable power of $\sim 11.6 \mathrm{~mW}$. To the authors' knowledge, this linewidth is one of the finest values reported in the literature on a single external cavity SIL-ECDL system. Lastly, both MRS and HRS configurations are found to be very stable at extreme operation conditions of high injection current and temperature when examined for a long-term stability period of $120 \mathrm{~min}$.

\section{Experimental Setup}

The schematic diagram of the MRS, along with the laboratory setup photograph, is shown in Figs. 1(a) and 1(b), respectively. A LD driver (Thorlabs, ITC4005) with built-in temperature control is used on a temperature-controlled LD mount (Thorlabs, TCLDM9) to drive the high-power commercially available Fabry Perot (FP) TO-can blue LDs (SN-LD-P06, SaNoor Technologies, and PL TB450B, Osram Licht AG) corresponding to MRS-1 and MRS-2, respectively. Both LDs exhibited a typical emission wavelength of $\sim 450 \mathrm{~nm}$. For perfect collimation without spherical aberration in the collimated beam, an aspheric lens (Thorlabs, A110TM-A) L1 with a focal length of $6.24 \mathrm{~mm}$ is used to collimate the free-running laser beam. The collimated beam then traverses through an equilateral dispersive prism (Thorlabs, PS858) $\mathrm{P}$ and then on to a 70:30 partially reflecting mirror (PR) (Edmund Optics, \#47-608 polka dot beam splitter) that is placed on a kinematic mount (Thorlabs, KM100). Hence, an external cavity is formed between the partially reflecting mirror and the front facet of the LD mount, with the dispersive prism in between, as shown in Fig. 1(a). The 70\% reflected beam of the laser is then fed back into the LD's active region via the front facet of the LD mount, while the transmitted $30 \%$ serves as the usable power of the system. The system power is then measured via a benchtop power meter (Newport, 2936-R) and, for spectral analysis, is then passed via a planoconvex lens (Thorlabs, LA-1951-A) L2 and focused on a fiber coupler to couple the power into a fiber that is then connected to an optical spectrum analyzer (Yokogawa, AQ6373B) with $20 \mathrm{pm}$ resolution. For the comprehensive analysis of the MRS that exhibits an estimated injection ratio (IR) of $\sim-1.5 \mathrm{~dB}$, three injection currents of 130,260 , and $390 \mathrm{~mA}$, two external cavity lengths of 22 and $16 \mathrm{~cm}$, and three heat-sink temperatures of $20^{\circ} \mathrm{C}, 30^{\circ} \mathrm{C}$, and $40^{\circ} \mathrm{C}$ are chosen. Later, a comprehensive comparison of the MRS configuration with our recently reported ${ }^{16}$ LRS and HRS systems, which corresponds to an estimated IR of $\sim-0.7$ and $\sim-3 \mathrm{~dB}$, respectively, have been performed in terms of tunability, $\Delta \lambda$, and SMSR. Finally, engaging identical setup and operating conditions of the HRS, here we performed a long-term stability analysis at high injection currents and high temperature on HRS and compared it with the MRS counterpart.
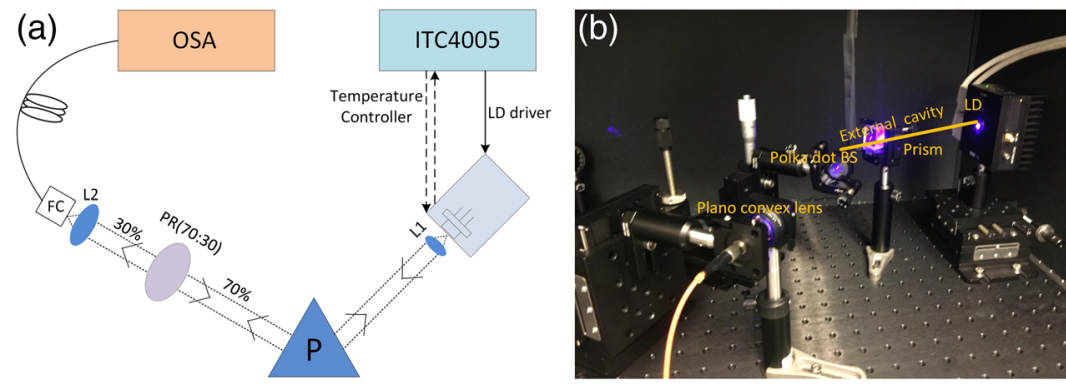

Fig. 1 (a) Schematic block diagram and (b) corresponding photograph of the laboratory setup of the MRS. For MRS-1 and MRS-2, the employed blue LDs are from SaNoor Technologies and Osram Licht AG, respectively. 

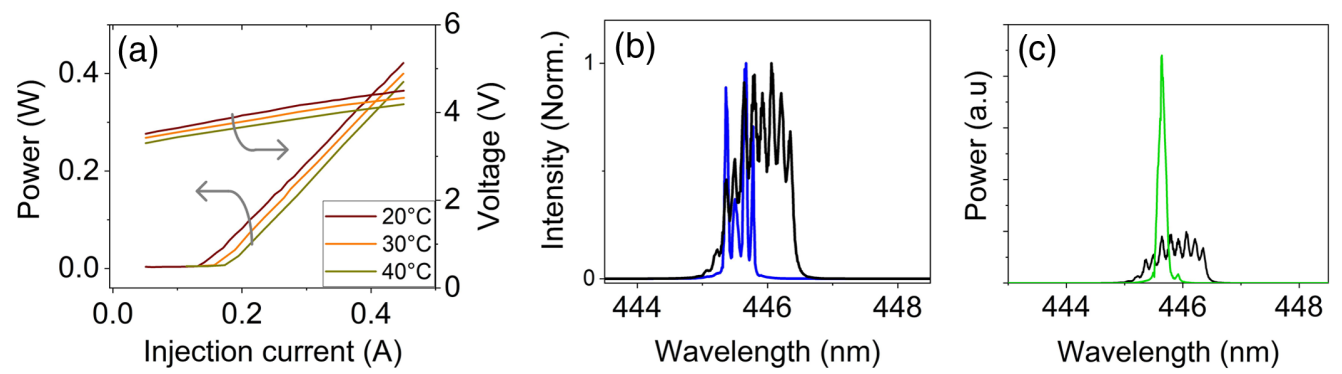

Fig. 2 (a) L-I-V characteristics of the free-running blue LD of MRS-1 at different temperatures, (b) the free-running lasing spectra of MRS-2 at $260 \mathrm{~mA}$ (blue) and $390 \mathrm{~mA}$ (black) at $20^{\circ} \mathrm{C}$. (c) Comparison between the free-running lasing (black) and the SIL locked mode (green) spectra at a high injection current of $390 \mathrm{~mA}$ and $20^{\circ} \mathrm{C}$, for MRS-2 configuration. In the case of (a), the freerunning optical power at $20^{\circ} \mathrm{C}$ is measured while at $30^{\circ} \mathrm{C}$ and $40^{\circ} \mathrm{C}$, the values are estimated from the LD datasheet.

\section{Self-Injection Locking Enhancements}

Figure 2(a) shows the optical power-injection current-voltage $(L-I-V)$ behavior of the MRS-1 LD at different heat-sink temperatures. At room temperature operation $\left(20^{\circ} \mathrm{C}\right)$, the device exhibited a lasing threshold current of about $125 \mathrm{~mA}$ with a slope efficiency of about $0.9 \mathrm{~W} / \mathrm{A}$. Moreover, the MRS-2 LD displayed a similar threshold current value but with higher slope efficiency (not shown here), suggesting a better quality active region device, and its free-running lasing spectra at injection currents of 260 and $390 \mathrm{~mA}$ are plotted in Fig. 2(b). More details of MRS-2 LD L-I-V characteristics can be obtained from Ref. 18. A clear multiple longitudinal modes emission is apparent with optical linewidths $\Delta \lambda$ (measured as the full-width at halfmaximum) of $\sim 450$ and $\sim 900 \mathrm{pm}$, respectively. In our prism-based ECDL system, we exploited an assistive self-injection locking scheme to achieve a phase-matching condition between the optical feedback mode and a free-running LD longitudinal mode for effective locking of that particular single mode that exists in the composite cavity (i.e., LD cavity and the external cavity). This is realized via fine-tuning the partially reflecting mirror position, hence altering the external cavity length, and attaining the phase-matching condition of the desired FP mode, thereby attaining wavelength tenability. ${ }^{16}$ In addition, the prism, which slightly disperses the feedback light into the LD's active region, further assists in achieving efficient locking of individual modes that are fundamentally dictated by the active region gain profile of the LD. A quasicontinuous wavelength tunability is accomplished with a step size equivalent to the LD mode spacing of $\sim 50 \mathrm{pm}$. The key signature of effective locking of a single-mode within the composite cavity is the reduction in the threshold current (due to decreased gain threshold) under optical feedback, ${ }^{19}$ which has been observed in this work. As a result, this resonant mode of the composite cavity will attain an earlier lasing condition, thereby consolidating all of the optical power while suppressing the adjoining modes. This is shown for the MRS-2 configuration in Fig. 2(c) with a near single locked mode at $445.63 \mathrm{~nm}$ alongside the free-running spectrum. There is a dramatic improvement in the spectral purity of the locked mode with a reduced -3 -dB optical bandwidth or $\Delta \lambda$ of $\sim 143 \mathrm{pm}$ compared with $\sim 900 \mathrm{pm}$ for the free-running counterpart, corresponding to an $\sim 6.3$ times improvement. In addition, this dominance facilitates reaching a high SMSR of $\sim 31 \mathrm{~dB}$ as well as a signal-to-noise ratio that could further improve the modulation bandwidth of the system. ${ }^{20}$ In the subsequent section, a rigorous analysis of the MRS is performed at two external cavity lengths of 22 and $16 \mathrm{~cm}$, three different heat-sink temperatures of $20^{\circ} \mathrm{C}, 30^{\circ} \mathrm{C}$, and $40^{\circ} \mathrm{C}$, and three injections currents of 130 (low), 260 (medium), and $390 \mathrm{~mA}$ (high).

\section{Medium Reflection System}

First, the MRS is comprehensively analyzed in this section and then compared with the other system configurations (i.e., LRS and HRS) to shed light on the effect of IR on the system performance. 


\subsection{Effect of Injection Current}

The performance of the MRS-1 configuration under three different heat sink temperatures of $20^{\circ} \mathrm{C}, 30^{\circ} \mathrm{C}$, and $40^{\circ} \mathrm{C}$ is shown in Fig. 3 at low, medium, and high injection currents and an external cavity length of $22 \mathrm{~cm}$, and the results are summarized in Table 1. At room temperature of $20^{\circ} \mathrm{C}$ [Fig. 3(a)], a tuning window of $\sim 8.2 \mathrm{~nm}(443.26$ to $451.46 \mathrm{~nm})$ at $130 \mathrm{~mA}$ is noted while the tunability range drops at 260 and $390 \mathrm{~mA}$, reaching values of $\sim 6.0$ and $\sim 5.6 \mathrm{~nm}$, respectively. This apparent decrease in the tuning window is ascribed to the intense mode competition at elevated injection currents due to an increased linewidth enhancement factor ( $\alpha$-factor) that degrades the phase-matching condition and hence the locking efficiency. This behavior was also observed not only in SIL tunable systems ${ }^{16,17}$ but also in other tunable ECDL systems such as Littrow and Littman configurations. ${ }^{12,14}$ Consequently, this has translated into inferior SMSR and $\Delta \lambda$ values with increasing injection current, as shown in Fig. 3(a) and Table 1. For instance, the medium and high injection current scenarios resulted in SMSR of $\sim 10$ to $27 \mathrm{~dB}$ and $\sim 8$ to $30 \mathrm{~dB}$, respectively, and $\Delta \lambda$ value of $\sim 80$ to $160 \mathrm{pm}$ and $\sim 140$ to $170 \mathrm{pm}$, respectively, while exhibiting mean SMSR of $\sim 18.5$ and $\sim 19 \mathrm{~dB}$, respectively, and mean $\Delta \lambda$ of $\sim 120$ and $\sim 155 \mathrm{pm}$, respectively. On the other hand, the characteristics at low injection current are significantly superior with SMSR and $\Delta \lambda$ values of $\sim 12$ to $33 \mathrm{~dB}$ (average $\sim 22.5 \mathrm{~dB}$ ) and $\sim 70$ to $90 \mathrm{pm}$ (average $\sim 80 \mathrm{pm}$ ), respectively, due to the efficient SIL, which allows for exploitation of the entire gain-bandwidth of the LD active region. Nevertheless, as observed from Table 1, a direct relation of the usable system power is noted with the current injection. While the free-running LD power at the front facet exhibited values of $\sim 3.0, \sim 183$, and $\sim 400 \mathrm{~mW}$ at 130, 260, and $390 \mathrm{~mA}$, respectively, the system under the locking condition measured usable powers (i.e., 30\% of PR) with corresponding values of $\sim 7.6, \sim 53$, and $\sim 110 \mathrm{~mW}$. Hence, these powers were employed to depict the respective injection current

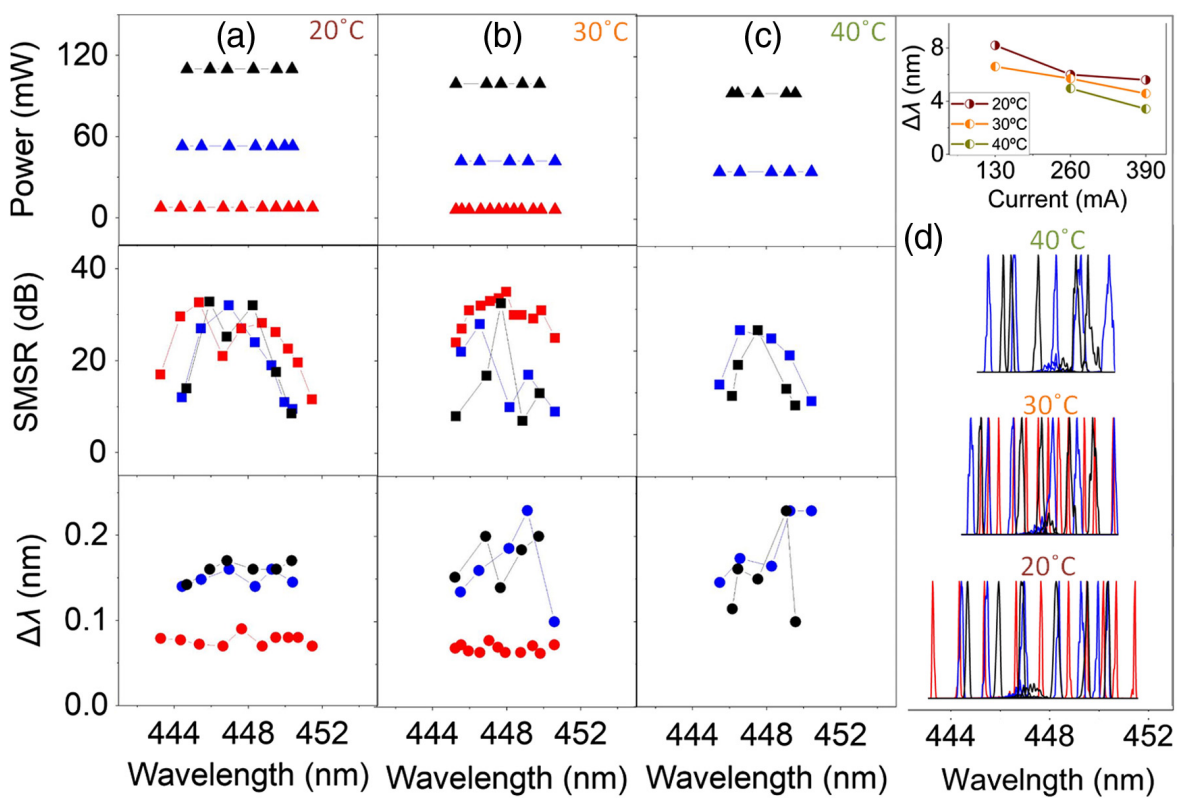

Fig. 3 Performance characteristics of MRS-1 in terms of wavelength tunability versus system power (triangles), SMSR (squares), and linewidth (circles) at (a) $20^{\circ} \mathrm{C}$, (b) $30^{\circ} \mathrm{C}$, and (c) $40^{\circ} \mathrm{C}$, and an external cavity length of $22 \mathrm{~cm}$. (d) The superimposed normalized SIL modes across the tuning window at different injection currents and different temperatures and the inset summarizes the measured tunability of MRS-1 as a function of injection currents at different temperatures. The red, blue, and black color markers correspond to the three injections of 130, 260, and $390 \mathrm{~mA}$, respectively. Note that the measured system power around the central SIL mode is extrapolated across the tuning window in (a), and the estimated system power from Fig. 2(a) is extrapolated across the respective tuning windows in (b) and (c). 
Table 1 Performance characteristics of MRS-1 configuration under different operating conditions.

\begin{tabular}{lccccc}
\hline \hline Temp. $\left({ }^{\circ} \mathrm{C}\right)$ & Current $(\mathrm{mA})$ & Tunability $(\mathrm{nm})$ & Power $(\mathrm{mW})$ & SMSR $(\mathrm{dB})$ & $\Delta \lambda(\mathrm{pm})$ \\
\hline 20 & 130 & 8.2 & 7.6 & 12 to $33(\sim 22)$ & 70 to $90(\sim 80)$ \\
& 260 & 5.6 & 53 & 10 to $27(\sim 18)$ & 80 to $160(\sim 120)$ \\
& 390 & 6 & 110 & 8 to $30(19)$ & 140 to $170(\sim 155)$ \\
30 & 130 & 6.6 & $6.2^{\mathrm{a}}$ & 24 to $35(\sim 29)$ & 63 to $78(\sim 71)$ \\
& 260 & 5.7 & $42^{\mathrm{a}}$ & 9 to $28(\sim 18)$ & 100 to $230(\sim 165)$ \\
40 & 390 & 4.6 & $99^{\mathrm{a}}$ & 7 to $32(\sim 19)$ & 140 to $200(\sim 170)$ \\
& 260 & 4.9 & $35^{\mathrm{a}}$ & 8 to $25(\sim 16)$ & 146 to $230(\sim 188)$ \\
& 390 & 3.4 & $93^{\mathrm{a}}$ & 7 to $25(16)$ & 100 to $230(\sim 165)$ \\
\hline \hline
\end{tabular}

The numbers inside the parentheses in the last two columns correspond to the average value.

${ }^{a}$ Estimated system power from Fig. 2(a).

wavelength tunability in Fig. 3(a) assuming similar power exhibited by all tuned modes across the wavelength tuning window. This suggests that the MRS configuration, with medium $\sim-1.5 \mathrm{~dB}$ IR (measured after L1), offers a larger tuning window with relatively higher system power compared with our very recently reported higher $(\sim-0.7 \mathrm{~dB})$ and lower $(\sim-3.0 \mathrm{~dB})$ IRs corresponding to the HRS and LRS configurations where either tunability or system power was observed to dominate. ${ }^{16}$

\subsection{Effect of Temperature}

Next, the effect of temperature on the MRS-1 performance is evaluated by increasing the heat sink temperature to $30^{\circ} \mathrm{C}$ and $40^{\circ} \mathrm{C}$. In this case, at $260 \mathrm{~mA}$, the corresponding optical powers, estimated from the LD datasheet, dropped by an average of $\sim 2 \%$ and $\sim 51 \%$, along with shrinking of wavelength tunability by a mean of $\sim 5.5 \%$ and $\sim 21 \%$, compared with the room temperature of $20^{\circ} \mathrm{C}$. The calculated system power at low, medium, and high injection currents, for the $30(40)^{\circ} \mathrm{C}$ is $\sim 6.2(-), \sim 42(\sim 35)$, and $\sim 99(\sim 93) \mathrm{mW}$, respectively, with exhibited tuning window of $\sim 6.6(-), \sim 5.7(\sim 4.9)$, and $\sim 4.6(\sim 3.4) \mathrm{nm}$, respectively, as shown in Figs. 3(b) and 3(c) and summarized in Table 1. It is to be noted that no lasing action was observed at $130 \mathrm{~mA}$ owing to the increase in the threshold current at $40^{\circ} \mathrm{C}$. Although the inferior system power is attributed to increased intrinsic losses of the quantum-well active region at elevated temperatures, thus deteriorating the slope efficiency and the lasing threshold current of the device, the shrinking of the tuning window is ascribed to difficulty in locking the modes that reside on the edges of the gain spectrum due to more poor phase-matching conditions owing to reduced gain. Nevertheless, the system's SMSR $(\Delta \lambda)$ showed a slight deterioration at elevated temperatures, as observed from Table 1, with a measured mean value of $\sim 29(\sim 71), \sim 18(\sim 165)$, and $\sim 19 \mathrm{~dB}(\sim 165 \mathrm{pm})$ at 130,260 , and $390 \mathrm{~mA}$, respectively, considering both $30^{\circ} \mathrm{C}$ and $40^{\circ} \mathrm{C}$. This translates to a mere $\sim 1 \mathrm{~dB}(\sim 0 \mathrm{pm})$ deterioration in SMSR $(\Delta \lambda)$ values when compared with room temperature for the medium and high injection current cases. Due to the deployment of the dispersive prism within the external cavity, which assisted slightly in refracting the competitive modes of the feedback beam at high injection current and temperature, the phase-matching condition and successful locking in this case were achieved compared with our previous design of SIL-ECDL systems reported in Ref. 17. On the other hand, comparing the SMSR $(\Delta \lambda)$ at low injection current between $20^{\circ} \mathrm{C}$ and $30^{\circ} \mathrm{C}$ in Table 1 , the latter demonstrates superior performance with a corresponding difference of $\sim 7 \mathrm{~dB}(\sim 9 \mathrm{pm})$ between the two, which may be due to very fine-tuning of the external cavity length manually. Hence, the deployment of piezo-driven motor stages should show a much better performance compared with the reported results of the SIL-ECDL system. 

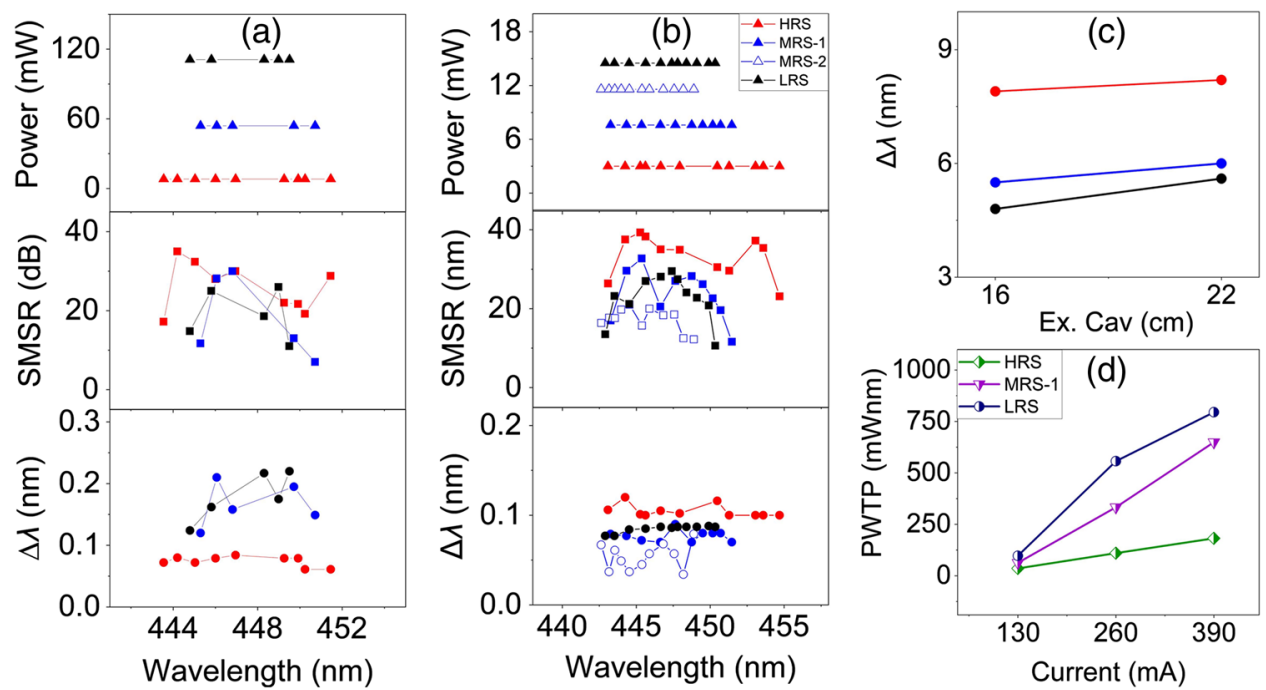

Fig. 4 (a) Performance characteristics of MRS-1 in terms of wavelength tunability versus system power (triangles), SMSR (squares), and linewidth (circles) at $20^{\circ} \mathrm{C}$, and an external cavity length of $16 \mathrm{~cm}$. Effect of IR, in the form of HRS, MRS-1, MRS-2, and LRS, on their respective performance, at a low injection current of $130 \mathrm{~mA}$ and $20^{\circ} \mathrm{C}$ and an external cavity length of $22 \mathrm{~cm}$. (c) Summary of the wavelength tunability of MRS-1 at different external cavity lengths, and (d) the system power wavelength tunability product at different injection currents as a function of IR, i.e., for HRS, MRS-1, and LRS. Note that the measured system power around the central SIL mode is extrapolated across the tuning window in (a) and (b) except for the MRS-2, where the usable power of the system is estimated from the respective LD datasheet ${ }^{18}$ and extrapolated across the tuning window. The red, blue, and black color markers correspond to the three injections of 130,260 , and $390 \mathrm{~mA}$, respectively.

\subsection{Effect of Cavity Length}

Then, to assess the effect of the external cavity length on the MRS- 1 configuration performance, the cavity is reduced by moving the PR $10 \mathrm{~cm}$ toward the dispersive prism, thereby exhibiting a value of $16 \mathrm{~cm}$. The performance was evaluated at low, medium, and high injection currents at $20^{\circ} \mathrm{C}$, and the extracted wavelength tunability, SMSR, and $\Delta \lambda$ values are plotted in Fig. 4(a). As reported earlier in our works, ${ }^{16,17}$ there is a direct relationship between the tuning window and the external cavity length of the SIL tunable system. For instance, at $130 \mathrm{~mA}$, the 22 -cm cavity demonstrated a wavelength tuning range of $\sim 8.2 \mathrm{~nm}$ while the 16-cm cavity showed $\sim 7.9 \mathrm{~nm}$ with a $\sim 0.3-\mathrm{nm}$ drop in value, with equivalent SMSR $(\Delta \lambda)$ of $\sim 12$ to $33 \mathrm{~dB}(\sim 70$ to $90 \mathrm{pm})$ and $\sim 17$ to $35 \mathrm{~dB}$ ( $\sim 61$ to $85 \mathrm{pm}$ ), respectively. Hence, at low injection, the locked mode characteristics are similar for both cavity lengths except that the SMSR curve is jagged, which could be due to difficulty in manually locking the modes. Furthermore, a similar drop in tuning window with an increase in cavity length is noted at medium and high injection currents. For instance, the tuning window at $260(390) \mathrm{mA}$ is reduced from $\sim 6.0(\sim 5.6) \mathrm{nm}$ to $\sim 5.5(\sim 4.8) \mathrm{nm}$, as summarized in Fig. 4(c), while the SMSR $\sim 7$ to $30(\sim 11$ to $26 \mathrm{~dB})$ and $\Delta \lambda \sim 120$ to 210 ( $\sim 162$ to $220 \mathrm{pm}$ ) unusually followed that of the $22 \mathrm{~cm}$ cavity with not much deterioration in the exhibited values. This is unlike the typical trend of inferior characteristics from external cavity LD systems in which the decreasing cavity length resulted from a more chaotic system in terms of laser active region dynamics. Hence, the results substantiate the performance preservation and the robustness of the system, which in essence can be attributed to the deployment of the dispersive prism within the external cavity, thereby allowing the modes to be slightly dispersed and providing efficient locking within the more chaotic system as well.

\section{Comparison of HRS, MRS, and LRS Configurations}

Here, we investigate the effect of optical feedback, or in other terms, IR, on the performance of the prism-based blue SIL-ECDL tunable system at a fixed temperature of $20^{\circ} \mathrm{C}, 22-\mathrm{cm}$ external 
Table 2 Summary of the comparative analysis of the three different IR systems at $130 \mathrm{~mA}$ and $20^{\circ} \mathrm{C}$.

\begin{tabular}{lccccc}
\hline \hline System & Tunability $(\mathrm{nm})$ & Power $(\mathrm{mW})$ & SMSR $(\mathrm{dB})$ & $\Delta \lambda(\mathrm{pm})$ & Ref. \\
\hline HRS & 12.11 & 3 & 23 to $40(\sim 31)$ & 100 to $110(\sim 105)$ & 16 \\
MRS-1 & 8.2 & 7.6 & 12 to $33(\sim 22)$ & 70 to $90(\sim 80)$ & This work \\
MRS-2 & 6.3 & $11.6^{\mathrm{a}}$ & 12 to $21(\sim 16)$ & 37 to $79(\sim 58)$ & This work \\
LRS & 7 & 15 & 15 to $30(\sim 22)$ & 77 to $88(\sim 83)$ & 16 \\
\hline \hline
\end{tabular}

The numbers inside the parentheses correspond to the average value.

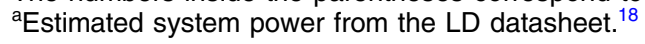

cavity length, and low injection current of $130 \mathrm{~mA}$. This is accomplished by varying the \% reflection of the PR to exhibit two more IR variations in addition to the $\sim-1.5 \mathrm{~dB}$ MRS-1 configuration, i.e., $\sim-0.7$ and $\sim-3 \mathrm{~dB}$ dubbed the LRS and HRS systems, respectively. ${ }^{16}$ Moreover, an additional MRS-2 configuration is also included in the comparison to shed light on the performance variation of the MRS system due to the deployment of different InGaN/GaN blue LDs. The summary of the results is shown in Fig. 4(b) in addition to tabulation in Table 2 for ease of comparison. Performance degradation in terms of wavelength tuning window and SMSR is observed while $\Delta \lambda$ is improved on increasing the IR. For example, the HRS showcases a wideband tuning window of $\sim 12.1 \mathrm{~nm}$, while the value decreased to $\sim 8.2$ and $\sim 7.0 \mathrm{~nm}$ for the MRS-1 and LRS, respectively, as shown in Fig. 4(b). In addition, upon increasing the injection current to 260 (390) mA (results not shown), a similar direct relationship between the tuning window and IR is noted, with the HRS, MRS-1, and LRS tuning spans further dropping to $\sim 8.3$ $(\sim 7.6) \mathrm{nm}, \sim 6.0(\sim 5.6) \mathrm{nm}$, and $\sim 6(\sim 4.5) \mathrm{nm}$, respectively. This observation is attributed to the increased amount of optical feedback power (i.e., increased IR), thereby further increasing the number of modes phase-matching condition and assisting in wideband tuning by efficiently locking the modes at the extreme edges of the laser active region gain profile. On the other hand, the LRS configuration with comparatively low feedback power sustains fewer external cavity modes to satisfy the phase-matching condition, which hinders the possibility of full exploitation of the laser active region gain profile.

Upon comparing the SMSR, $\Delta \lambda$, and usable system power from Table 2 at low injection current, HRS, due to its high IR, exhibits broader $\Delta \lambda$ with relatively higher SMSR compared with LRS and MRS- 1 configurations, which displayed $\Delta \lambda<100$ pm but with decreased SMSR values. The narrow $\Delta \lambda$ from MRS and LRS compared with the HRS configuration as well as other SIL-ECDL systems, ${ }^{17}$ and similar to that of grating-based systems, ${ }^{11,14,21}$ is attributed to the reduced number of cavity modes solution for the phase matching condition and thus efficient locking. On the other hand, inferior SMSR is ascribed to the reduced threshold gain difference before and after locking the mode and hence a diminished optical power difference with respect to adjacent modes. In terms of the system power, at $130 \mathrm{~mA}$, HRS exhibited the lowest value of $\sim 3 \mathrm{~mW}$ compared with LRS, which demonstrated $\sim 15 \mathrm{~mW}$, while MRS-1 settled at a reasonable value of $\sim 7.6 \mathrm{~mW}$, as shown in Table 2 . In addition, the interplay between the tuning window and the usable optical power across the three configurations (i.e., at different IRs) is compared in the form of system power-wavelength tunability product (PWTP) and plotted in Fig. 4(d) at $20^{\circ} \mathrm{C}$ and 22-cm external cavity length. While on average, the low injection current demonstrated a similar PWTP of $\sim 65 \mathrm{~mW} \mathrm{~nm}$ from all three configurations, the value substantially departs at medium and high injection currents, with LRS demonstrating the largest value of $\sim 676 \mathrm{~mW} \mathrm{~nm}$ followed by $\sim 491 \mathrm{~mW} \mathrm{~nm}$ by MRS-1 and then $\sim 146 \mathrm{~mW} \mathrm{~nm}$ by HRS. This suggests that MRS configuration is an optimum choice with reasonable PWTP for practical application, exhibiting both relatively broader wavelength tunability as well as system power simultaneously.

Furthermore, another MRS system with a different blue LD (Osram Licht AG, PL TB450B) is also investigated here (MRS-2); its performance, particularly slope efficiency, is better than the LD of MRS-1. The room temperature and low injection current results, which are plotted in 
Fig. 4(b) along with MRS-1, demonstrate a significantly reduced $\Delta \lambda$ (by an average of $\sim 28 \%$ ) exhibited by the system, measuring $\sim 37$ to $79 \mathrm{pm}$ with an average of $\sim 58 \mathrm{pm}$, and improved estimated usable power of $\sim 11.6 \mathrm{~mW}$, as shown in Table 2 . However, the wavelength tunability and the SMSR deteriorate with measured values of $\sim 6.3 \mathrm{~nm}(442.62$ to $448.9 \mathrm{~nm})$ and $\sim 12$ to $21 \mathrm{~dB}$ (mean value of $\sim 16 \mathrm{~dB}$ ), respectively, which may be due to the optimized narrow gain profile of the LD-active region, hence limiting the tuning window. Nonetheless, the measured $\Delta \lambda$ from MRS-2, to the authors' knowledge, is the best value reported in the literature on a single external cavity-based SIL-ECDL system and is comparable to that of the grating-assisted ECDL systems.

\section{System Stability}

To affirm the suitability of MRS configuration for practical applications due to its reasonable PWTP and other performance characteristics, a thorough stability analysis in terms of both short(30 min) and long (120 min) periods is performed at critical conditions of high injection currents as well as high temperatures. Moreover, to further strengthen the prism-based SIL-ECDL system prospects in practical deployments, the HRS configuration, which corresponds to the highest IR and is expected to be the most vulnerable system in terms of active region dynamics, is employed to analyze its stability features; these are discussed in the subsequent section.

\subsection{MRS Configuration}

Short-term stability analysis of the MRS-1 and MRS-2 is performed under two respective injection currents of 130 and $390 \mathrm{~mA}$ and corresponding to the locked mode wavelength of 445.64 and $446.84 \mathrm{~nm}$ at an interval of $3 \mathrm{~min}$. The extracted optical power, $\Delta \lambda$, SMSR, and peak wavelength $(\lambda)$ at a fixed cavity length of $22 \mathrm{~cm}$ and $20^{\circ} \mathrm{C}$ are plotted in Fig. 5(a), and the summarized performance fluctuations are shown in Table 3. As seen, the peak $\lambda$ and $\Delta \lambda$ showed a minor fluctuation of $\sim \pm 1.5 \mathrm{pm}$ for both systems, while the SMSR and the power of the MRS-1 demonstrated a slight variation in the range of $\pm 0.35 \mathrm{~dB}$ and $\pm 0.13 \mathrm{~dB}$, respectively. On the other
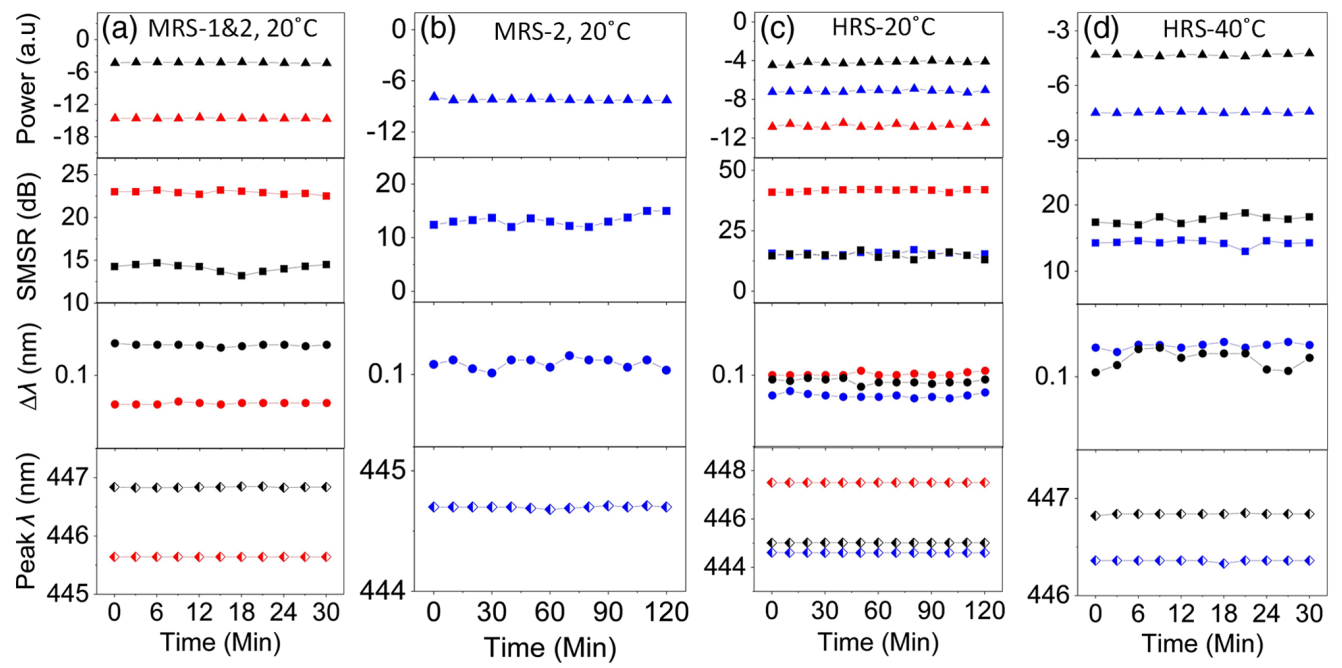

Fig. 5 Stability analysis of the prism-based tunable blue laser system at different IRs, in terms of (a) and (b) MRS, and (c) and (d) HRS configurations while monitoring the integrated power (triangles), SMSR (squares), linewidth (circles), and peak wavelength (half-closed circles). Short-term 30 min stability analysis of (a) MRS-1 and MRS-2 at 130 and $390 \mathrm{~mA}$, respectively, at $20^{\circ} \mathrm{C}$ and (d) $\mathrm{HRS}$ at 260 and $390 \mathrm{~mA}$ at $40^{\circ} \mathrm{C}$. Long-term 120 min stability analysis of (b) MRS-2 at $260 \mathrm{~mA}$ and (c) $\mathrm{HRS}$ at 130,260 , and $390 \mathrm{~mA}$ at $20^{\circ} \mathrm{C}$. In all cases, the external cavity length is fixed at $22 \mathrm{~cm}$. In all cases, red, blue, and black markers correspond to injection current of 130 , 260 , and $390 \mathrm{~mA}$, respectively. 
Table 3 Summarized stability performance of the three IR systems under different operating conditions.

\begin{tabular}{|c|c|c|c|c|c|c|c|c|}
\hline System & Temp. $\left({ }^{\circ} \mathrm{C}\right)$ & Current (mA) & Time (min) & SMSR (dB) & Power (dB) & $\Delta \lambda(\mathrm{pm})$ & Peak $\lambda(\mathrm{pm})$ & Ref \\
\hline \multirow[t]{5}{*}{ HRS } & \multirow[t]{3}{*}{20} & 130 & 120 & \pm 0.62 & \pm 0.4 & \pm 1.5 & \pm 1.5 & \multirow[t]{5}{*}{ This work } \\
\hline & & 260 & & \pm 1.3 & \pm 0.42 & \pm 2.5 & \pm 3.5 & \\
\hline & & 390 & & \pm 2 & \pm 0.42 & \pm 3 & \pm 5 & \\
\hline & \multirow[t]{2}{*}{40} & 260 & \multirow[t]{2}{*}{30} & \pm 1 & \pm 0.1 & \pm 3.5 & \pm 14 & \\
\hline & & 390 & & \pm 2 & \pm 0.2 & \pm 8 & \pm 8 & \\
\hline MRS-1 & 20 & 130 & 30 & \pm 0.35 & \pm 0.13 & \pm 1.5 & \pm 1.5 & This work \\
\hline \multirow[t]{2}{*}{ MRS-2 } & \multirow[t]{2}{*}{20} & 390 & 30 & \pm 1.5 & \pm 0.2 & \pm 1.5 & \pm 1.5 & \multirow[t]{2}{*}{ This work } \\
\hline & & 260 & 120 & \pm 0.15 & \pm 1.5 & \pm 6 & \pm 10 & \\
\hline LRS & 20 & 130 & 30 & \pm 0.3 & \pm 0.3 & \pm 3 & \pm 5 & 16 \\
\hline
\end{tabular}

hand, MRS-2, which operates at a higher injection current of $390 \mathrm{~mA}$, demonstrated similar features to that of MRS-1 at a low injection current except in the SMSR, which is more than twice in value.

Moreover, long-term stability of MRS-2 is also conducted at a medium $260 \mathrm{~mA}$ injection current at $20^{\circ} \mathrm{C}$ and an interval of $10 \mathrm{~min}$, and the extracted performance parameters are plotted in Fig. 5(b) and tabulated in Table 3. As noticed, the fluctuation in the performance variables increased in general when monitored for an extended period. In this case, the peak $\lambda$ exhibited a variation of $\pm 10 \mathrm{pm}, \Delta \lambda$ (SMSR) showed a shift in the range of $\pm 6 \mathrm{pm}( \pm 1.5 \mathrm{~dB})$, and the mode power showed a slight variation of about $\pm 0.2 \mathrm{~dB}$. In general, the performance of MRS-2 under the stringent operating condition of medium/high injection current can be considered acceptable.

\subsection{HRS Configuration}

After that, HRS configuration is considered, and a short-term stability analysis is performed at medium and high injection currents at $40^{\circ} \mathrm{C}$ temperature, and the data are collected at an interval of $3 \mathrm{~min}$. The extracted values at a fixed $22 \mathrm{~cm}$ external cavity length are summarized in Fig. 5(d) and in Table 3, where the peak $\lambda$ showed fluctuations of about \pm 8 to $14 \mathrm{pm}$, in general, at $\geq 260 \mathrm{~mA}$. On the one hand, measured $\Delta \lambda$ and the SMSR at $260 \mathrm{~mA}$ exhibited about $\pm 3.5 \mathrm{pm}$ and $\pm 1.0 \mathrm{~dB}$ fluctuation, respectively, while the mode power showed a minor variation of about $\pm 0.1 \mathrm{~dB}$ throughout the monitoring window. On the other hand, at $390 \mathrm{~mA}$, which is an extreme operating condition considering the operating temperature, the $\Delta \lambda$ (SMSR) and mode power showcased a fluctuation of about $\pm 8 \mathrm{pm}(\sim 2 \mathrm{~dB})$ and about $\sim 0.2 \mathrm{~dB}$, respectively.

In addition, the stability of the HRS configuration is further ascertained by performing a 120-min long-term analysis with a time step of $10 \mathrm{~min}$. The plots of the extracted parameter values are summarized in Fig. 5(c), and the variations are given in Table 3. As noted, at low injection current, the peak $\lambda$ shows the least fluctuation, followed by medium injection current, and then high injection current, showing a direct effect of locking efficiency degradation with increases in the current injection. The corresponding $\Delta \lambda$ (SMSR) exhibits a variation of $\pm 1.5 \mathrm{pm}( \pm 0.62 \mathrm{~dB}), \pm 2.5 \mathrm{pm}( \pm 1.3 \mathrm{~dB})$, and $\pm 3 \mathrm{pm}( \pm 2 \mathrm{~dB})$ for the entire monitoring time, while the respective mode power showed close variation at all three injection currents, as shown in Table 3. In summary, comparing across the prism-based SIL-ECDL system at three IR reveals similar and remarkable stability even at extreme operating conditions, thus reinstating the robustness of the proposed system and strengthening its potential for a plethora of applications. 


\section{Conclusion}

In summary, a rigorous investigation of MRS configuration and the effects of IR and temperature on the stability of a prism-based SIL quasicontinuously tunable InGaN/GaN blue semiconductor LD system are discussed. The analysis of this MRS configuration showcased simultaneous system power and wavelength tunability performance optimization while maintaining excellent $\Delta \lambda$ and SMSR compared with HRS and LRS configurations due to the observed direct (inverse) relationship of the IR and the tuning window (usable system power). In addition, investigation of two MRS configurations based on two distinct commercial LDs was conducted at an injection current of $130 \mathrm{~mA}$, where a quasicontinuous wavelength tunability of $\sim 8.2 \mathrm{~nm}$, average $\Delta \lambda$ of $\sim 80 \mathrm{pm}$, and SMSR of $\geq 12 \mathrm{~dB}$ were achived from the MRS-1 configuration while exhibiting a usable power of $\sim 7.6 \mathrm{~mW}$. In contrast, the MRS-2 configuration exhibited a reduced wavelength tuning range of $\sim 6.3 \mathrm{~nm}$ with a mean $\Delta \lambda$ of $\sim 58 \mathrm{pm}$, SMSR of $\geq 12 \mathrm{~dB}$, and estimated system power of $\sim 11.6 \mathrm{~mW}$. Moreover, stringent stability assessment of the two prism-based SIL-ECDL systems, namely MRS and HRS, at different operating conditions strengthens the high stability features of the proposed systems even at extreme operation conditions and thus substantiate the potential deployment of the systems in different fields of application.

\section{Acknowledgments}

The authors (SM and MZMK) would like to thank King Fahd University of Petroleum and Minerals for supporting this research and acknowledge support from King Abdulaziz City for Science and Technology (KACST), Technology Innovation Center (TIC) for Solid-State Lighting sub-awarded grant EE002381 and the main grant KACST TIC R2-FP-08. The authors (JAH, OA, IA, TKN, and BSO) acknowledge support from King Abdullah University of Science and Technology (KAUST) through baseline funding BAS/1/1614-01-01 and KAUST-KFUPM Special Initiative program (REP/1/2878-01-01).

\section{References}

1. K. Holc et al., "Blue laser diodes for trace matter detection," Opt. Appl. 40(3), 641-651 (2010).

2. Y. Shimada et al., "A simplified 461-nm laser system using blue laser diodes and a hollow cathode lamp for laser cooling of Sr," Rev. Sci. Instrum. 84(6), 063101 (2013).

3. I. S. Burns, J. Hult, and C. F. Kaminski, "Spectroscopic use of a novel blue diode laser in a wavelength region around $450 \mathrm{~nm}$, , Appl. Phys. B Lasers Opt. 79(4), 491-495 (2004).

4. A. Müller et al., "Diode laser based light sources for biomedical applications," Laser Photonics Rev. 7(5), 605-627 (2013).

5. N. Ruhnke et al., "Single-pass UV generation at $222.5 \mathrm{~nm}$ based on high-power GaN external cavity diode laser," Opt. Lett. 40(9), 2127 (2015).

6. T. Tanaka et al., "Littrow-type external-cavity blue laser for holographic data storage," Appl. Opt. 46(17), 3583-3592 (2007).

7. L. Coldren, M. Mashanovitch, and S. W. Corzine, Diode Lasers and Photonic Integrated Circuits, 2nd ed., John Wiley \& Sons, Inc., Hoboken, New Jersey (2012).

8. J. H. Kang et al., "DFB laser diodes based on GaN using 10th order laterally coupled surface gratings," IEEE Photonics Technol. Lett. 30(3), 231-234 (2018).

9. Z. Deng et al., "InGaN/GaN distributed feedback laser diodes with surface gratings and sidewall gratings," Micromachines 10(10), 1-10 (2019).

10. J. A. Holguin-Lerma et al., "480-nm distributed-feedback InGaN laser diode for 105-Gbit/s visible-light communication," Opt. Lett. 45(3), 742 (2020).

11. N. Ruhnke et al., "400 $\mathrm{mW}$ external cavity diode laser with narrowband emission at 445 nm," Opt. Lett. 39(13), 3794 (2014).

12. D. Ding et al., "Tunable high-power blue external cavity semiconductor laser," Opt. Laser Technol. 94, 1-5 (2017). 
13. M. Chi, O. B. Jensen, and P. M. Petersen, "Tuning range and output power optimization of an external-cavity GaN diode laser at 455 nm," Appl. Opt. 55(9), 2263 (2016).

14. D. Ding et al., "Influence of grating parameters on the performance of a high-power blue external-cavity semiconductor laser," Appl. Opt. 57(7), 1589 (2018).

15. M. H. Chen et al., "Single longitudinal mode external cavity blue InGaN diode laser," Opt. Laser Technol. 116(March), 68-71 (2019).

16. S. Mukhtar et al., "Blue laser diode system with an enhanced wavelength tuning range," IEEE Photonics J. 12(2), 1-10 (2020).

17. M. H. M. Shamim et al., "Tunable self-injection locked green laser diode," Opt. Lett. 43(20), 4931 (2018).

18. Osram Licht AG, "PL-TB450B laser diode datasheet," https://dammedia.osram.info/media/ resource/hires/osram-dam-7159367/PLTB450B_EN.pdf (accessed 16 June 2020).

19. J. Ohtsubo, Semiconductor Lasers, 4th ed., Springer International Publishing, Switzerland (2013).

20. M. H. M. Shamim et al., "Investigation of self-injection locked visible laser diodes for high bit-rate visible light communication," IEEE Photonics J. 10(4), 7905611 (2018).

21. M. Chi et al., "Tunable high-power external-cavity gan diode laser systems in the visible spectral range," Chapter 1 in Laser Technology and Its Applications, Y. Ma, pp. 3-21, IntechOpen Limited, London, United Kingdom (2019).

Biographies of the authors are not available. 